associated with fruit and vegetable intake were included. Random effects models were used to estimate summary RRs.

Results Nineteen cohort studies were included in the meta-analysis. The summary RR for the highest vs the lowest intake was 0.92 (95\% CI 0.86 to 0.99 ) for fruit and vegetables combined, $0.90(95 \%$ CI 0.83 to 0.98 ) for fruit and 0.91 ( $95 \%$ CI 0.86 to 0.96 ) for vegetables. The inverse associations appear to be restricted to colon cancer. In linear dose-response analysis only intake of vegetables was significantly associated with colorectal cancer risk, summary RR 0.98 (95\% CI 0.97 to 0.99 ) per $100 \mathrm{~g}$ per day. However, significant inverse associations emerged in non-linear models for fruits ( $p_{\text {non- }}$ linearity $<0.001)$ and vegetables $\left(p_{\text {non-linearity }}=0.001\right)$. The greatest risk reduction was observed when increasing intake from very low levels of intake. There was generally little evidence of heterogeneity in the analyses and there was no evidence of small-study bias.

Conclusion This meta-analysis indicates that there is a weak, but statistically significant non-linear inverse association between fruit and vegetable intake and colorectal cancer risk.

\section{3-3.5 \\ REVISITING THE RISK OF COELIAC DISEASE IN CHILDREN BORN SMALL FOR GESTATIONAL AGE: A OUASI-EXPERIMENTAL FAMILY-BASED APPROACH}

doi:10.1136/jech.2011.142976a.95

C J Wingren,* D Agardh, J Merlo. Lund University, Faculty of Medicine, Malmö, Skåne, Sweden

Introduction Celiac disease (CD) is a chronic small bowel disease with a strong heritability. Several perinatal conditions are today considered as risk factors for $\mathrm{CD}$ in children. One of these conditions is being born small for gestational age (SGA). However, if the association between SGA and CD risk in children is causal is unknown. Therefore, we aimed to apply a quasi-experimental family based (OEFB) design that is a powerful strategy for studying causal relationships. Methods Using the Swedish Medical Birth Registry linked to a number of other national databases we identified all singleton children born in Sweden between 1987 and 1993 ( $n=781624$ ). We applied a OEFB design, and compared sibling with discrepant exposure (ie, being or not SGA) in relation to their risk of CD from birth until they were 2-year old. We also performed classical logistic regression analyses adjusting for known risk factors for $\mathrm{CD}$ but without the OEFB design.

Results In the classical adjusted logistic regression analysis, we found an association between being SGA and CD risk: OR 1.34 95\% CI 1.03 to 1.74. However when applying the OEFB design and conditional logistic regression this association disappeared: OR 1.05 95\% CI 0.53 to 2.06 .

Conclusion Our results suggest that previous finding indicating an association between being SGA and CD risk were confounded. These previous results might be explained by the fact that the offspring from mothers with CD are frequently SGA and, because the strong heritability, they have also a higher risk for CD.

\section{3-3.6 FOOD PATTERNS AND ALL-CAUSE MORTALITY AMONG ADULTS AGED >65 YEARS: A COMPARISON OF METHODS}

doi:10.1136/jech.2011.142976a.96

${ }^{1} \mathrm{~S}$ McNaughton, ${ }^{*} \mathrm{C}$ Bates, ${ }^{3} \mathrm{G}$ Mishra. ${ }^{1}$ Centre for Physical Activity and Nutrition Research, School of Exercise and Nutrition Sciences, Deakin University, Melbourne,
Victoria, Australia; ${ }^{2}$ MRC Human Nutrition Research, Cambridge, UK; ${ }^{3}$ School of Population Health, University of Queensland, Brisbane, Queensland, Australia

Introduction Analysis of dietary patterns offers an alternative approach to the investigation of diet and health. The aim of this study was to compare different approaches to assessing dietary patterns in the same cohort, and their associations with all-cause mortality.

Methods Analysis was based on 972 participants of the British Diet and Nutrition Survey of adults aged $>65$ years in 1994/1995 who were followed-up for mortality status until 2008. We examined the generalisability of reduced rank regression (RRR) methods from a previous study of all-cause mortality using: (1) an RRR-derived dietary pattern (known as the "Unhealthy Eating Index-UHI"; high in red meat, added fat, potatoes, refined grains, processed meat; low in fruit) and (2) dietary patterns derived from exploratory RRR using BMI, total cholesterol, HDL cholesterol, triglycerides and blood pressure as the intermediate markers. Three measures of diet quality, the Healthy Diet Score, the Recommended Food Score (RFS) and the Mediterranean Diet Score (MDS) were also investigated. Cox proportional hazards regression was conducted using follow-up time as the time variable.

Results After adjustment for potential confounders, the MDS, the RFS and the UHI remained associated with mortality (highest vs lowest quartile; MDS HR 0.77, 95\% CI, 0.61 to 0.97; RFS HR 0.67, $95 \%$ CI 0.52 to 0.86 ; UHI HR 1.14 , $95 \%$ CI 1.09 to 1.82 ). No significant associations were shown for the Healthy Diet Score or any of the exploratory RRR dietary patterns.

Conclusion Not all dietary patterns were associated with all-cause mortality. Further work is needed to test the generalisabilty of dietary patterns across cohorts.

\subsection{THE EPIDEMIOLOGY OF COGNITIVE RESERVE IN AGEING}

\section{Chair: Dr. Fiona Matthews, UK 03-4.1 COGNITIVE LIFESTYLE, DEMENTIA PROTECTION AND
THE BRAIN'S RESERVE MECHANISMS}

doi:10.1136/jech.2011.142976a.97

M Valenzuela.* University of New South Wales, Sydney, Australia

Cognitive lifestyle refers to acquired patterns of cognitive and mental activity over the course of one's lifetime, and in our group has been formalised to include information about educational achievement, occupational complexity, social engagement and cognitively-loaded leisure activities. Many large-scale prospective cohort studies have shown that maintaining a more active cognitive lifestyle is linked with a reduction in incident dementia risk. Recently, we have further shown that the combination of a higher educational level with either a more cognitively challenging job in mid-life, or enhanced social activity in later life, is more important for minimising dementia risk than either factor in isolation.

Brain reserve and cognitive reserve are typically suggested to mediate this protective relationship. In this presentation, a brief history of the terms will be given, as well as a deconstruction into more specific biological mechanisms. An evaluation of the role of disease modifying, neuroprotective and compensatory mechanisms is then possible, and will be discussed in relation to the design of clinical trials and community-based studies. 